\begin{tabular}{|c|l|}
\hline Title & Non-relativistic Limit of a Dirac Polaron in Relativistic Quantum Electrodynamics \\
\hline Author(s) & A rai, A sao \\
\hline Citation & $\begin{array}{l}\text { Letters in Mathematical Physics, 77/3), 283-290 } \\
\text { https:/doi.org/40.1007/311005-006-0098-y }\end{array}$ \\
\hline Issue Date & 2006 \\
\hline Doc URL & http://hdl.handle.net/2115/14748 \\
\hline Rights & The original publication is available at www.springerlink.com \\
\hline Type & article (author version) \\
\hline File Information & LMP2006-77-3.pdf \\
\hline
\end{tabular}

Instructions for use 


\title{
Non-relativistic Limit of a Dirac Polaron in Relativistic Quantum Electrodynamics
}

\author{
Asao Arai* \\ Department of Mathematics, Hokkaido University \\ Sapporo 060-0810, Japan \\ E-mail: arai@math.sci.hokudai.ac.jp
}

\begin{abstract}
A quantum system of a Dirac particle interacting with the quantum radiation field is considered in the case where no external potentials exist. Then the total momentum of the system is conserved and the total Hamiltonian is unitarily equivalent to the direct integral $\int_{\mathbf{R}^{3}}^{\oplus} \overline{H(\mathbf{p})} d \mathbf{p}$ of a family of self-adjoint operators $\overline{H(\mathbf{p})}$ acting in the Hilbert space $\oplus^{4} \mathcal{F}_{\text {rad }}$, where $\mathcal{F}_{\text {rad }}$ is the Hilbert space of the quantum radiation field. The fibre operator $\overline{H(\mathbf{p})}$ is called the Hamiltonian of the Dirac polaron with total momentum $\mathbf{p} \in \mathbf{R}^{3}$. The main result of this paper is concerned with the non-relativistic (scaling) limit of $\overline{H(\mathbf{p})}$. It is proven that the non-relativistic limit of $\overline{H(\mathbf{p})}$ yields a self-adjoint extension of a Hamiltonian of a polaron with spin $1 / 2$ in non-relativistic quantum electrodynamics.
\end{abstract}

Mathematics Subject Classifications (2000): 81V10, 81Q10, 47N50

Keywords: quantum electrodynamics, polaron, Dirac-Maxwell operator, non-relativistic limit, scalig limit, Fock space

\section{Introduction}

In a previous paper [2], the author discussed the non-relativistic (scaling) limit of a particle-field Hamiltonian $H$, called a Dirac-Maxwell (DM) operator or a DM Hamiltonian, in relativistic quantum electrodynamics (QED). The Hamiltonian $H$ describes a Dirac particle - a relativistic charged particle with spin $1 / 2$ - in an external potential $V$ and interacting with the quantum radiation field. In the case where $V=0$, the total momentum of the quantum system is conserved, implying that $H$ is unitarily equivalent

${ }^{*}$ Supported by the Grant-in-Aid No.17340032 for Scientific Research from the JSPS. 
to a self-adjoint operator $\widetilde{H}$ which has a direct integral decomposition $\widetilde{H}=\int_{\mathbf{R}^{3}}^{\oplus} \overline{H(\mathbf{p})} d \mathbf{p}$ with $H(\mathbf{p})$ being an essentially self-adjoint operator acting in the Hilbert space

$$
\mathcal{H}:=\mathbf{C}^{4} \otimes \mathcal{F}_{\text {rad }}=\oplus^{4} \mathcal{F}_{\text {rad }}
$$

$(\overline{H(\mathbf{p})}$ denotes the closure of $H(\mathbf{p}))$, where

$$
\mathcal{F}_{\text {rad }}:=\oplus_{n=0}^{\infty} \otimes_{\mathrm{s}}^{n}\left[L^{2}\left(\mathbf{R}^{3}\right) \oplus L^{2}\left(\mathbf{R}^{3}\right)\right]
$$

is the Fock space over $L^{2}\left(\mathbf{R}^{3}\right) \oplus L^{2}\left(\mathbf{R}^{3}\right)$ ( $\otimes_{\mathrm{s}}^{n}$ denotes $n$-fold symmetric tensor prodct and $\left.\otimes_{\mathrm{s}}^{0}\left[L^{2}\left(\mathbf{R}^{3}\right) \oplus L^{2}\left(\mathbf{R}^{3}\right)\right]:=\mathbf{C}\right)$, that is, the Hilbert space of state vectors for the quantum radiation field. The 3 -dimensional vector $\mathbf{p} \in \mathbf{R}^{3}$ physically means a point in the spectrum of the total momentum [1, Theorem 1.6]. The operator $H(\mathbf{p})$ describes a Dirac polaron, namely, the Dirac particle coupled to the quantum radiation field with the total momentum equal to $\mathbf{p}$. Based on this picture, we call the fiber operator $H(\mathbf{p})$ the Hamiltonian of the Dirac polaron with total momentum p.

In this paper we consider a partial non-relativistic limit of $H(\mathbf{p})$, where "partial" means that non-relativistic limit is taken only with respect to the Dirac particle. For this purpose, in a way similar to that done in [2], we put a scaling parameter $\kappa>0$ into $H(\mathbf{p})$ and make an energy cutoff for the free Hamiltonian of the quantum radiation field as well as an energy renormalization to obtain a natural candidate $H_{\kappa}(\mathbf{p})$ on which the non-relativistic limit $\kappa \rightarrow \infty$ should be considered (the parameter $\kappa$ plays a role of the speed of light). We show that the resolvent $\left(\overline{H_{\kappa}(\mathbf{p})}-z\right)^{-1}(z \in \mathbf{C} \backslash \mathbf{R})$ strongly converges to an operator matrix, one of the components of which is the resolvent of a self-adjoint extension of the Hamiltonian of the non-relativistic polaron with spin $1 / 2$ in non-relativistic QED. This is the main result of this paper.

In Section 2 we describe some basic facts on both relativistic and non-relativistic polarons in QED. In Section 3 we precisely formulate the main result and prove it.

\section{Preliminaries}

\subsection{The explicit form of the operator $H(\mathbf{p})$}

We first recall some objects in relativistic QED. We use a unit system in which $\hbar$ (the Planck constant divided by $2 \pi$ ) and $c$ (the speed of light) are equal to 1 respectively.

We denote by $\alpha_{j}, \beta(j=1,2,3)$ the Dirac matrices, i.e., $4 \times 4$-Hermitian matrices satisfying the anticommutation relations

$$
\left\{\alpha_{j}, \alpha_{l}\right\}=2 \delta_{j l} 1_{4}, \quad\left\{\alpha_{j}, \beta\right\}=0, \quad j, l=1,2,3, \quad \beta^{2}=1_{4},
$$

where $\{X, Y\}:=X Y+Y X$ and $1_{4}$ is the $4 \times 4$-identity matrix. We set $\boldsymbol{\alpha}:=\left(\alpha_{1}, \alpha_{2}, \alpha_{3}\right)$. In the present paper we take the following representations of $\alpha_{j}$ and $\beta$, called the standard representation $[7, \mathrm{p} .36]$ :

$$
\alpha_{j}=\left(\begin{array}{cc}
0_{2} & \sigma_{j} \\
\sigma_{j} & 0_{2}
\end{array}\right), \quad \beta=\left(\begin{array}{cc}
1_{2} & 0_{2} \\
0_{2} & -1_{2}
\end{array}\right), \quad j=1,2,3,
$$


where $1_{2}\left(\right.$ resp. $\left.0_{2}\right)$ is the $2 \times 2$-identity (resp. zero) matrix and $\sigma_{j}$ 's are the Pauli matrices:

$$
\sigma_{1}:=\left(\begin{array}{cc}
0 & 1 \\
1 & 0
\end{array}\right), \quad \sigma_{2}:=\left(\begin{array}{cc}
0 & -i \\
i & 0
\end{array}\right), \quad \sigma_{3}:=\left(\begin{array}{cc}
1 & 0 \\
0 & -1
\end{array}\right) .
$$

Let $\omega: \mathbf{R}^{3} \rightarrow[0, \infty)$ be a Borel measurable function, strictly positive a.e. (almost everywhere) with respect to the Lebesgue measure. For mathematical generality, we take one-particle (one-photon) Hamiltonian to be the multiplication operator on $L^{2}\left(\mathbf{R}^{3}\right) \oplus$ $L^{2}\left(\mathbf{R}^{3}\right)$ by the function $\omega$; we denote it by the same symbol $\omega$. Then the free Hamiltonian of the quantum radiation field is defined by

$$
H_{\mathrm{rad}}:=d \Gamma(\omega),
$$

the second quantization of $\omega([4, \mathrm{p} .302],[5, \S \mathrm{X} .7])$. The momentum operator

$$
\mathbf{P}_{\text {rad }}=\left(P_{\text {rad }, 1}, P_{\text {rad }, 2}, P_{\text {rad }, 3}\right)
$$

of the quantum radiation field is given by

$$
P_{\mathrm{rad}, j}:=d \Gamma\left(k_{j}\right), \quad j=1,2,3,
$$

the second quantization of $k_{j}$ as the multiplication operator by the $j$-th coordinate function $k_{j}$ in the momentum space $\mathbf{R}^{3}$ of a photon.

We denote by $a(f)\left(f \in L^{2}\left(\mathbf{R}^{3}\right) \oplus L^{2}\left(\mathbf{R}^{3}\right)\right)$ the annihilation operator acting in $\mathcal{F}_{\text {rad }}$ and define

$$
\phi(f):=\frac{a(f)+a(f)^{*}}{\sqrt{2}},
$$

the Segal field operator on $\mathcal{F}_{\text {rad }}[5, \S \mathrm{X} .7]$.

There exist $\mathbf{R}^{3}$-valued continuous functions $\mathbf{e}^{(r)}, r=1,2$, on the nonsimply connected space $\mathbf{M}_{0}:=\mathbf{R}^{3} \backslash\left\{\left(0,0, k_{3}\right) \mid k_{3} \in \mathbf{R}\right\}$ such that

$$
\mathbf{e}^{(r)}(\mathbf{k}) \cdot \mathbf{e}^{(s)}(\mathbf{k})=\delta_{r s}, \quad \mathbf{e}^{(r)}(\mathbf{k}) \cdot \mathbf{k}=0, \quad r, s=1,2, \mathbf{k} \in \mathbf{M}_{0} .
$$

We set $\mathbf{e}^{(r)}\left(0,0, k_{3}\right)=0, k_{3} \in \mathbf{R}$. The vector-valued functions $\mathbf{e}^{(r)}, r=1,2$, describe the polarization of one photon.

Let $g \in L^{2}\left(\mathbf{R}^{3}\right)$ and

$$
g_{j}:=\left(g e_{j}^{(1)}, g e_{j}^{(2)}\right) \in L^{2}\left(\mathbf{R}^{3}\right) \oplus L^{2}\left(\mathbf{R}^{3}\right), \quad j=1,2,3 .
$$

We define a smeared quantum radiation field $\mathbf{A}:=\left(A_{1}, A_{2}, A_{3}\right)$ by

$$
A_{j}:=\phi\left(g_{j}\right), \quad j=1,2,3
$$

We assume the following:

(g.1) $\omega g, g / \sqrt{\omega},|\mathbf{k}| g,|\mathbf{k}| g / \sqrt{\omega} \in L^{2}\left(\mathbf{R}^{3}\right)$.

(g.2) For all $R>0, \int_{|\mathbf{k}| \leq R} \omega(\mathbf{k})^{2} d \mathbf{k}<\infty$ (i.e., $\omega \in L_{\text {loc }}^{2}\left(\mathbf{R}^{3}\right)$ ).

For objects $\mathbf{a}=\left(a_{1}, a_{2}, a_{3}\right)$ and $\mathbf{b}=\left(b_{1}, b_{2}, b_{3}\right)$ such that the product $a_{j} b_{j}$ and the sum $\sum_{j=1}^{3} a_{j} b_{j}$ are defined, we write $\mathbf{a} \cdot \mathbf{b}:=\sum_{j=1}^{3} a_{j} b_{j}$.

Now we can give the explicit form of the polaron Hamiltonian $H(\mathbf{p})\left(\mathbf{p} \in \mathbf{R}^{3}\right)$ [1]:

$$
H(\mathbf{p})=\boldsymbol{\alpha} \cdot\left(\mathbf{p}-\mathbf{P}_{\mathrm{rad}}-q \mathbf{A}\right)+m \beta+H_{\mathrm{rad}},
$$

where $q \in \mathbf{R}$ (resp. $m>0$ ) is a constant, physically denoting the charge (resp. bare mass) of the Dirac particle under consideration. 


\subsection{Essential self-adjointness}

Let $\Omega_{0}:=\{1,0,0, \cdots\}$ be the Fock vacuum in $\mathcal{F}_{\text {rad }}$ and $\mathcal{F}_{\text {rad }, 0}^{\infty}$ be the subspace algebraically spanned by the vectors $\Omega_{0}, a\left(f_{1}\right)^{*} \cdots a\left(f_{n}\right)^{*} \Omega_{0}, n \in \mathbf{N}, f_{j} \in C_{0}^{\infty}\left(\mathbf{R}^{3}\right) \oplus C_{0}^{\infty}\left(\mathbf{R}^{3}\right), j=$ $1, \cdots, n$. Then $\mathcal{F}_{\text {rad }, 0}^{\infty}$ is dense in $\mathcal{F}_{\text {rad }}$. We introduce

$$
\mathcal{D}:=\oplus^{4} \mathcal{F}_{\mathrm{rad}, 0}^{\infty}
$$

which is dense in $\mathcal{H}$. The following fact is proven [1, Theorem 1.5]:

Theorem 1 Assume (g.1) and (g.2). Then, for all $\mathbf{p} \in \mathbf{R}^{3}, H(\mathbf{p})$ is essentially selfadjoint on $\mathcal{D}$.

Remark 1 It is a highly non-trivial problem to make it clear whether or not $H(\mathbf{p})$ is bounded below, because $H(\mathbf{p})$ contains the term $-\boldsymbol{\alpha} \cdot \mathbf{P}_{\text {rad }}$ which is unbounded both below and above and "comparable" with $H_{\text {rad }}$. Recently Sasaki [6] gave a positive solution to this problem, proving that, under a suitable condition for $g, H(\mathbf{p})$ is bounded below for all $\mathbf{p} \in \mathbf{R}^{3}$ and $q \in \mathbf{R}$.

\subsection{A scaled Hamiltonian}

To consider the non-relativistic limit of $H(\mathbf{p})$, we put a scaling parameter $\kappa>0$ in $H(\mathbf{p})$ and make an energy cutoff for $H_{\text {rad }}$ as well as an energy renormalization. Let $\Lambda:[0, \infty) \rightarrow[0, \infty)$ be an increasing measurable function such that $\lim _{\kappa \rightarrow \infty} \Lambda(\kappa)=\infty$ and

$$
H_{\mathrm{rad}}^{(\kappa)}:=E_{\mathrm{rad}}([0, \Lambda(\kappa)]) H_{\mathrm{rad}} E_{\mathrm{rad}}([0, \Lambda(\kappa)]),
$$

where $E_{\mathrm{rad}}(\cdot)$ is the spectral measure of $H_{\mathrm{rad}}$. The operator $H_{\mathrm{rad}}^{(\kappa)}$ may be interpreted as the free Hamiltonian of the quantum radiation field with an energy cutoff. Then the scaled, renormalized polaron Hamiltonian for which we consider a scaling limit is defined as follows:

$$
H_{\kappa}(\mathbf{p}):=\kappa \boldsymbol{\alpha} \cdot\left(\mathbf{p}-\mathbf{P}_{\mathrm{rad}}-q \mathbf{A}\right)+m \kappa^{2} \beta-m \kappa^{2}+H_{\mathrm{rad}}^{(\kappa)} .
$$

The non-relativistic limit by which we mean in the present paper is to take the limit $\kappa \rightarrow \infty$ of $H_{\kappa}(\mathbf{p})$ in a suitable sense, where the scaling parameter $\kappa$ plays a role of the speed of light. As remarked in [2], this non-relativistic limit is with respect to the Dirac particle only and, hence, strictly speaking, it is a partial non-relativistic limit.

\subsection{A Dirac type operator}

In connection with the non-relativistic limit of $H_{\kappa}(\mathbf{p})$, we find it convenient to introduce a Dirac type operator defined by

$$
\not D_{\mathbf{A}}(\mathbf{p}):=\boldsymbol{\alpha} \cdot\left(\mathbf{p}-\mathbf{P}_{\mathrm{rad}}-q \mathbf{A}\right)
$$

(For a general background, see [2, Section 4].)

Theorem 2 Assume (g.1) and (g.2). Then, for all $\mathbf{p} \in \mathbf{R}^{3}, \not D_{\mathbf{A}}(\mathbf{p})$ is essentially selfadjoint on $\mathcal{D}$. 
Proof. It is sufficient to show that $-\boldsymbol{\alpha} \cdot\left(\mathbf{P}_{\text {rad }}+q \mathbf{A}\right)$ is essentially self-adjoint, since $\boldsymbol{\alpha} \cdot \mathbf{p}$ is a bounded self-adjoint operator. This is easily done by applying the commutator theorem [5, Theorem X.37] (see the proof of [1, Theorem 1.5]).

As a corollary to this theorem, we have the following fact:

Corollary 3 Assume (g.1) and (g.2). Then, for all $\kappa>0$ and $\mathbf{p} \in \mathbf{R}^{3}, H_{\kappa}(\mathbf{p})$ is essentially self-adjoint on $\mathcal{D}$.

Proof. The operator $H_{\kappa}(\mathbf{p})$ is written as $H_{\kappa}(\mathbf{p})=\kappa \not D_{\mathbf{A}}(\mathbf{p})+B$ with $B:=m \kappa^{2} \beta-$ $m \kappa^{2}+H_{\text {rad }}^{(\kappa)}$. Note that $H_{\text {rad }}^{(\kappa)}$ is a bounded self-adjoint operator. Hence $B$ is a bounded self-adjoint operator. Therefore, by Theorem 2 and the Kato-Rellich theorem, $H_{\kappa}(\mathbf{p})$ is essentially self-adjoint on $\mathcal{D}$.

Using the representation (2.2), we have for $\not_{\mathbf{A}}(\mathbf{p})$ the following operator matrix representation:

$$
\not D_{\mathbf{A}}(\mathbf{p})=\left(\begin{array}{cc}
0 & P_{\mathbf{A}}(\mathbf{p}) \\
P_{\mathbf{A}}(\mathbf{p}) & 0
\end{array}\right)
$$

with

$$
P_{\mathbf{A}}(\mathbf{p}):=\boldsymbol{\sigma} \cdot\left(\mathbf{p}-\mathbf{P}_{\mathrm{rad}}-q \mathbf{A}\right),
$$

where $\boldsymbol{\sigma}:=\left(\sigma_{1}, \sigma_{2}, \sigma_{3}\right)$. In the same way as in the case of $\not D_{\mathbf{A}}(\mathbf{p})$, we can prove the following theorem:

Theorem 4 Assume (g.1) and (g.2). Then, for all $\mathbf{p} \in \mathbf{R}^{3}, P_{\mathbf{A}}(\mathbf{p})$ is essentially selfadjoint on $\mathcal{E}:=\oplus^{2} \mathcal{F}_{\text {rad, } 0}^{\infty}$.

Theorems 2 and 4 imply the following operator equalities:

$$
\overline{D_{\mathbf{A}}(\mathbf{p})}=\left(\begin{array}{cc}
0 & \overline{P_{\mathbf{A}}(p)} \\
\overline{P_{\mathbf{A}}(\mathbf{p})} & 0
\end{array}\right)
$$

and

$$
{\overline{\not D_{\mathbf{A}}(\mathbf{p})}}^{2}=\left(\begin{array}{cc}
{\overline{P_{\mathbf{A}}(p)}}^{2} & 0 \\
0 & {\overline{P_{\mathbf{A}}(\mathbf{p})}}^{2}
\end{array}\right) .
$$

\subsection{A non-relativistic polaron}

The Hamiltonian (with total momentum $\mathbf{p} \in \mathbf{R}^{3}$ ) of a non-relativistic charged polaron with spin $1 / 2$ and with bare mass $m>0$ is defined by

$$
H_{\mathrm{NR}}(\mathbf{p}):=\frac{1}{2 m} P_{\mathbf{A}}(\mathbf{p})^{2}+H_{\mathrm{rad}}
$$

It can be shown that, for all sufficiently small $|q|, H_{\mathrm{NR}}(\mathbf{p})$ is essentially self-adjoint [3]. It seems, however, that the essential self-adjointness of $H_{\mathrm{NR}}(\mathbf{p})$ for all $q \in \mathbf{R}$ has not been proved in the literature. In fact, this is a non-trivial problem, because $H_{\mathrm{NR}}(\mathbf{p})$ contains the terms $\mathbf{P}_{\text {rad }}^{2}$ and $\mathbf{A} \cdot \mathbf{P}_{\text {rad }}$ which are not relatively bounded with respect to $H_{\text {rad }}$. But 
here we do not discuss this problem. Instead we construct a self-adjoint extension of $H_{\mathrm{NR}}(\mathbf{p})$.

For each $\kappa>0$ we introduce

$$
H_{\mathrm{NR}}^{(\kappa)}(\mathbf{p}):=\frac{1}{2 m}{\overline{P_{\mathbf{A}}(\mathbf{p})}}^{2}+H_{\mathrm{rad}}^{(\kappa)} .
$$

Since $H_{\mathrm{rad}}^{(\kappa)}$ is bounded as remarked above, it follows that $H_{\mathrm{NR}}^{(\kappa)}(\mathbf{p})$ is self-adjoint with $D\left(H_{\mathrm{NR}}^{(\kappa)}(\mathbf{p})\right)=D\left({\overline{P_{\mathbf{A}}(\mathbf{p})}}^{2}\right)$ and nonnegative. The following lemma is a key to the main result of this paper:

Lemma 5 Assume (g.1) and (g.2). Then there exists a unique self-adjoint extension $\tilde{H}_{\mathrm{NR}}(\mathbf{p})$ of $H_{\mathrm{NR}}(\mathbf{p})$ such that the following hold:

(i) $\tilde{H}_{\mathrm{NR}}(\mathbf{p}) \geq 0$.

(ii) $D\left(\tilde{H}_{\mathrm{NR}}(\mathbf{p})^{1 / 2}\right) \subset D\left(\left|\overline{P_{\mathbf{A}}(\mathbf{p})}\right|\right) \cap D\left(H_{\mathrm{rad}}^{1 / 2}\right)$.

(iii) For all $z \in \mathbf{C} \backslash[0, \infty)$,

$$
\text { S- } \lim _{\kappa \rightarrow \infty}\left(H_{\mathrm{NR}}^{(\kappa)}(\mathbf{p})-z\right)^{-1}=\left(\tilde{H}_{\mathrm{NR}}(\mathbf{p})-z\right)^{-1} .
$$

(iv) For all $\xi<0$ and $\psi \in D\left(\tilde{H}_{\mathrm{NR}}(\mathbf{p})^{1 / 2}\right)$,

$$
\text { S- } \lim _{\kappa \rightarrow \infty}\left(H_{\mathrm{NR}}^{(\kappa)}(\mathbf{p})-\xi\right)^{1 / 2} \psi=\left(\tilde{H}_{\mathrm{NR}}(\mathbf{p})-\xi\right)^{1 / 2} \psi \text {. }
$$

Proof. We need only to apply [2, Theorem A.1] to the following case:

$$
N=1, A=\frac{1}{2 m}{\overline{P_{\mathbf{A}}(\mathbf{p})}}^{2}, B_{1}=H_{\mathrm{rad}}
$$

where $N, A, B_{j}(j=1, \cdots, N)$ are the notations used in [2, Appendix A].

Remark 2 Since $H_{\mathrm{NR}}(\mathbf{p})$ is nonnegative, it has a self-adjoint extension $\hat{H}_{\mathrm{NR}}$ which is defined as the Friedrichs extension of $H_{\mathrm{NR}}$. Another self-adjoint extension $H_{\mathrm{NR}}^{\prime}$ of $H_{\mathrm{NR}}(\mathbf{p})$ can be defined through the sesquilinear form $s(\Psi, \Phi):=\sum_{j=1}^{3}\left\langle\overline{P_{\mathbf{A}}(\mathbf{p})_{j}} \Psi, \overline{P_{\mathbf{A}}(\mathbf{p})_{j}} \Phi\right\rangle / 2 m+$ $\left\langle H_{\mathrm{rad}}^{1 / 2} \Psi, H_{\mathrm{rad}}^{1 / 2} \Phi\right\rangle, \Psi, \Phi \in \cap_{j=1}^{3} D\left(\overline{P_{\mathbf{A}}(\mathbf{p})_{j}}\right) \cap D\left(H_{\mathrm{rad}}^{1 / 2}\right)$. But it seems to be nontrivial to see if $\hat{H}_{\mathrm{NR}}=H_{\mathrm{NR}}^{\prime}$. Unfortunately we have not been able to clarify if $\tilde{H}_{\mathrm{NR}}=\hat{H}_{\mathrm{NR}}$ or $\tilde{H}_{\mathrm{NR}}=H_{\mathrm{NR}}^{\prime}$. Also we remark that, if $H_{\mathrm{NR}}(\mathbf{p})$ is not essentially self-adjoint, then the operator $\tilde{H}_{\mathrm{NR}}(\mathbf{p})$ may depend on the choice of the energy cutoff parameter $\Lambda(\cdot)$. 


\section{The Main Result}

The main result of the present paper is as follows:

Theorem 6 Assume (g.1) and (g.2). Suppose that

$$
\lim _{\kappa \rightarrow \infty} \frac{\Lambda(\kappa)^{2}}{\kappa}=0
$$

Then, for all $z \in \mathbf{C} \backslash \mathbf{R}$ and all $\mathbf{p} \in \mathbf{R}^{3}$,

$$
\text { S- } \lim _{\kappa \rightarrow \infty}\left(\overline{H_{\kappa}(\mathbf{p})}-z\right)^{-1}=\left(\begin{array}{cc}
\left(\tilde{H}_{\mathrm{NR}}(\mathbf{p})-z\right)^{-1} & 0 \\
0 & 0
\end{array}\right) .
$$

Proof. It is easy to see that

$$
\overline{H_{\kappa}(\mathbf{p})}=\overline{\not D_{\mathbf{A}}(\mathbf{p})}+m \kappa^{2} \beta-m \kappa^{2}+H_{\mathrm{rad}}^{(\kappa)} .
$$

In the same way as in the proof of [2, Lemma 5.1], we can show that the self-adjoint operator $\overline{D_{\mathbf{A}}(\mathbf{p})}$ strongly anticommutes with $\beta$. Since we assume (3.1) and we have Lemma 5 , we can apply [2, Theorem 4.3$]$ to the following case:

$$
A=\overline{D_{\mathbf{A}}(\mathbf{p})}, \quad B=m \beta, \quad C(\kappa)=H_{\mathrm{rad}}^{(\kappa)},
$$

where $A, B$ and $C(\kappa)$ are the notations used in [2, Section 4]. Thus we obtain (3.2).

Theorem 6 establishes a natural connection of relativistic QED to non-relativistic QED in the context of polaron theory.

\section{References}

[1] A. Arai, A particle-field Hamiltonian in relativistic quantum electrodynamics, J. Math. Phys. 41 (2000), 4271-4283.

[2] A. Arai, Non-relativistic limit of a Dirac-Maxwell operator in relativistic quantum electrodynamics, Rev. Math. Phys. 15 (2003), 245-270.

[3] F. Hiroshima and H. Spohn, Ground state degeneracy of the Pauli-Fierz model with spin, Adv. Theor. Math. Phys. 5 (2001), 1091-1104.

[4] M. Reed and B. Simon, Methods of Modern Mathematical Physics I: Functional Analysis, Academic Press, New York, 1972.

[5] M. Reed and B. Simon, Methods of Modern Mathematical Physics II: Fourier Analysis, Self-adjointness, Academic Press, New York, 1975.

[6] I. Sasaki, Ground state energy of the polaron in the relativistic quantum electrodynamics, J. Math. Phys. 46 (2005), 102307.

[7] B. Thaller, The Dirac Equation, Springer-Verlag, Berlin, Heidelberg, 1992. 